\title{
BORDER EFFECT, FRACTAL STRUCTURES AND BRIGHTNESS OSCILLATIONS IN NON-SILVER PHOTOGRAPHIC MATERIALS
}

\author{
${ }^{1,2}$ A. B. Plachenov, ${ }^{3}$ B. T. Plachenov \\ ${ }^{1}$ Moscow State Technical University of Radioengineering, Electronics and Automation, \\ Moscow, Russia \\ ${ }^{2}$ Saint-Petersburg State University of Aerospace Instrumentation, St. Petersburg, Russia \\ ${ }^{3}$ Baltic State Technical University "VOENMEH" named after D. F. Ustinov, \\ St. Petersburg, Russia \\ a_plachenov@mail.ru,plachenov32@yandex.ru

\begin{abstract}
PACS 42.70.Gi, 42.70.Jk, 68.35.Fx, 68.35.bm, 68.37.-d, 68.55.A-, 68.55.Ac, 68.55.Ln, 68.55.am, 78.47.dc, 82.20.-w, 82.30.Cf, 82.30.Lp, 82.30.Nr, 82.33.Pt, 82.35.Np, 82.37.Vb, 82.40.-g, 82.40.Qt, 82.45.Mp, 82.50.-m, 82.56.Lz
\end{abstract}

DOI 10.17586/2220-8054-2015-6-1-133-139

\begin{abstract}
A brief review of effects in information recording systems based on complex compounds of polyvinyl alcohol (PVA) with metals $(\mathrm{Au}, \mathrm{Cu}, \mathrm{Pt}, \mathrm{Bi})$ is presented. As the result of irradiation, the chain reaction process of decomposition causes the aggregation of metal atoms or metal salts molecules to point centers or dendritic crystals. Some features of these processes are outlined.
\end{abstract}

Keywords: Non-silver photography, chain process, diffusion, aggregation, fractal dimension.

Received: 11 December 2014

Revised: 17 December 2014

\section{Introduction}

In this paper, we want to present a brief review of works performed in the 1980's - early 1990's, mainly at the Leningrad Mechanical Institute and Khabarovsk Pedagogical Institute. This research was devoted to the investigation of photoinitiated and radiationinitiated processes in thin films of polyvinyl alcohol (PVA) with complex ions of gold, copper, platinum, bismuth and other metals inserted into the polymer chain. These investigations were stimulated by fast depletion of silver supplies used in photomaterials. In these circumstances, the search for alternative media of photographic information recording seemed a very practical task. Now, the technological development in the field of the digital photography has radically reduced the severity of this problem. Nevertheless, in some fields the traditional method of image recording still remains preferable, and new materials based on results obtained in this research in question may appear useful. At the same time, we want to underline, that physical processes found in investigated materials, in our view, are of great interest themselves, as for some of these processes and we have no satisfactory explanation for some of these processes. We hope that this article will perhaps invite the attention of the contemporary generation of researchers to these questions. 


\section{Chain process of light-induced decomposition of complex ions of heavy metals}

As was established in [1-3], the light-induced (and also radiation-induced) decomposition of halogen-containing compounds of certain metals in PVA films, such as solid solution $H\left[\mathrm{AuCl}_{4}\right]-\mathrm{PVA}$, is carried out in accordance with a chain reaction process. The process is initiated by excitation of a halogen in the auric complex ion:

$$
\left[\mathrm{AuCl}_{4}\right]^{-}+h \nu \rightarrow\left\{\left[\mathrm{AuCl}_{4}\right]^{-}\right\}^{*} \rightarrow \mathrm{AuCl}+2 \mathrm{Cl} \cdot+\mathrm{Cl}^{-} \text {. }
$$

As a result of this primary reaction (1), we obtain chlorine radicals $C l \cdot$ which propagate along polymer chains and cause further decomposition of ions:

$$
\left[\mathrm{AuCl}_{4}\right]^{-}+\mathrm{Cl} \cdot \rightarrow \mathrm{AuCl}+\mathrm{Cl} \cdot+\mathrm{Cl}_{2}+\mathrm{Cl}^{-} .
$$

The chain continuation reaction (2) repeats over and over again. The chain length appears not less than $10^{3}$. This process can be facilitated by reactions between $\mathrm{Cl}$-radicals with PVA, these reactions also have chain character.

During propagation, the chlorine radicals can be captured by traps or two radicals can form the $C l_{2}$ molecule. These reactions lead to chain termination.

After decomposition of complex metal ions, some additional reactions can follow. Products of these reactions, metal atoms or salt molecules, diffuse and aggregate:

$$
\begin{gathered}
A u C l \rightarrow \text { phase } \\
3 A u C l \rightarrow 2 A u+A u C l_{3} \\
A u \rightarrow \text { phase }
\end{gathered}
$$

Analogous processes take place in PVA films containing complex ions of other metals.

\section{Bordering effect}

If the film is lightened uniformly, then metal or metal salts aggregate at point centers, which are also uniformly distributed, see Fig 1 . When the concentration of metal (or salt) increases, then the concentration of phase centers also increases, while at the same time, the sizes of centers decrease, because the probability of the center formation increases nonlinearly with metal concentration.

When the irradiation is not uniform, then, as a rule, the concentration of the precipitated metal (salt) increases with an increase in the irradiation density. Nevertheless, if we have a large difference in this density, so that it is very high in the light-affected area, and it is negligible in the shadow area, we can observe the additional enlargement of point centers near the boundary of these areas (border effect) [4], see Fig 2. At the same time, the concentration of point centers near the boundary remains approximately the same as inside the irradiated zone. There are also some rare, large centers in shadow area near the boundary.

To explain this effect, the following model was proposed. After high-intensity irradiation, almost all complex ions in irradiated area decompose, and atoms of metal (salt molecules) aggregate to phase centres with uniform size and concentration. At the same time, $C l^{*}$-radicals which appear in the primary reaction (1), move to the shadow zone and cause the secondary reaction (2) here. The metal (salt), which appears in this reaction, begins to diffuse. As the rate of the reaction (2) is rather small, then the concentration of metal (salt) is also small. Under these conditions, the majority of the metal do not form 


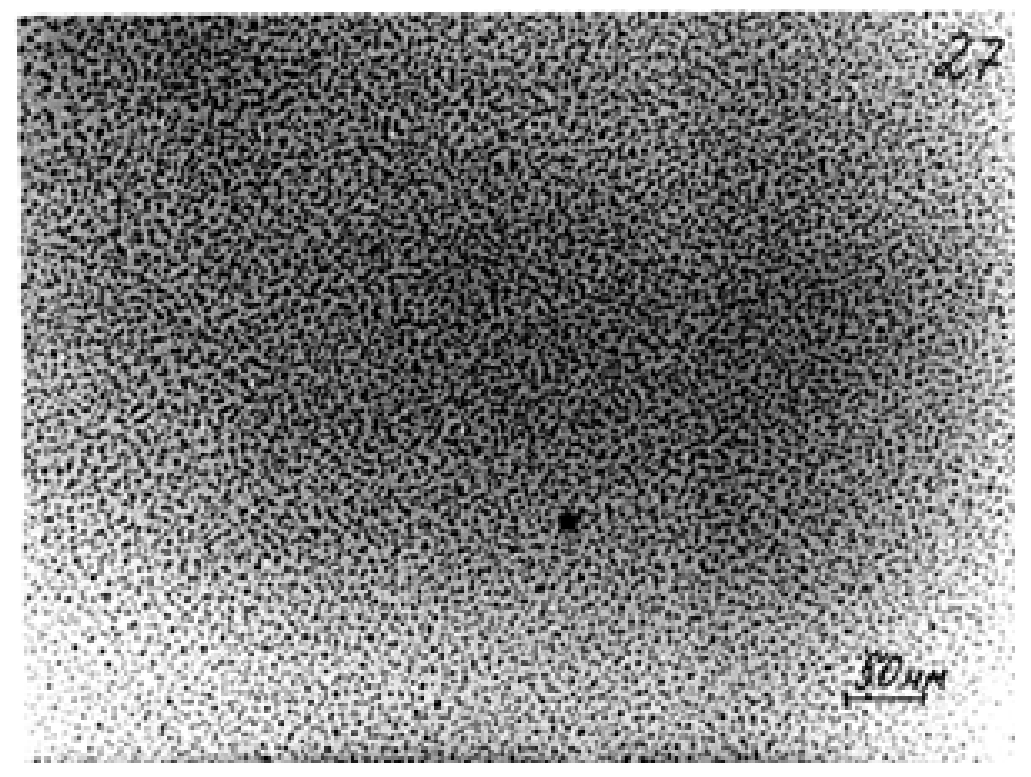

FIG. 1. Centres of $C u$-phase aggregation

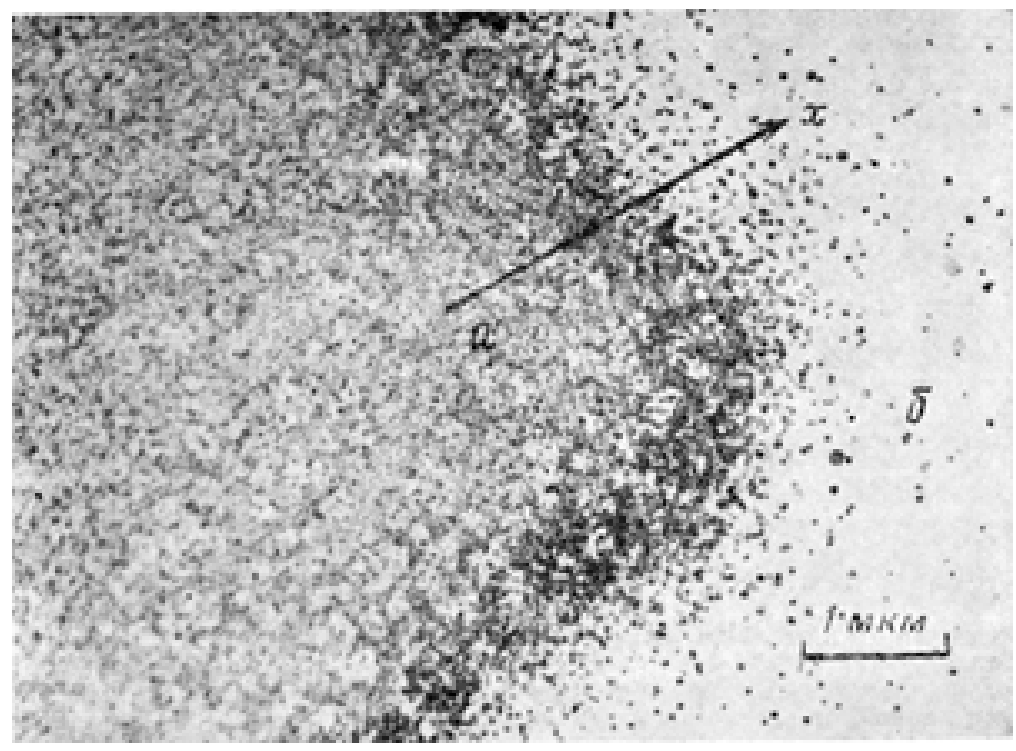

Fig. 2. Centers of $\mathrm{AuCl}$-phase aggregation near the boundary between lightened and shadow areas in the PVA-H $\left[\mathrm{AuCl}_{4}\right]$ film

centers in the shadow area, but diffuse to irradiated region and cause the growth of phase centers near the boundary.

To describe this process, a simplified mathematical model was proposed and explicit formulae were obtained. The results of calculations are in satisfactory agreement with experimental data.

\section{Fractal structures}

When the primary reaction is induced not by light, but by a high-energetic electron beam, then under some additional conditions (e.g., initial concentration of complex ions must belong to some diapason), then it can aggregate not to point centers, but to dendritic 
crystals [5]. Fig. 3 and 4 show that the growth of such crystals begins from the boundary of the film or from some impurities, e.g. point centers which were formed earlier.

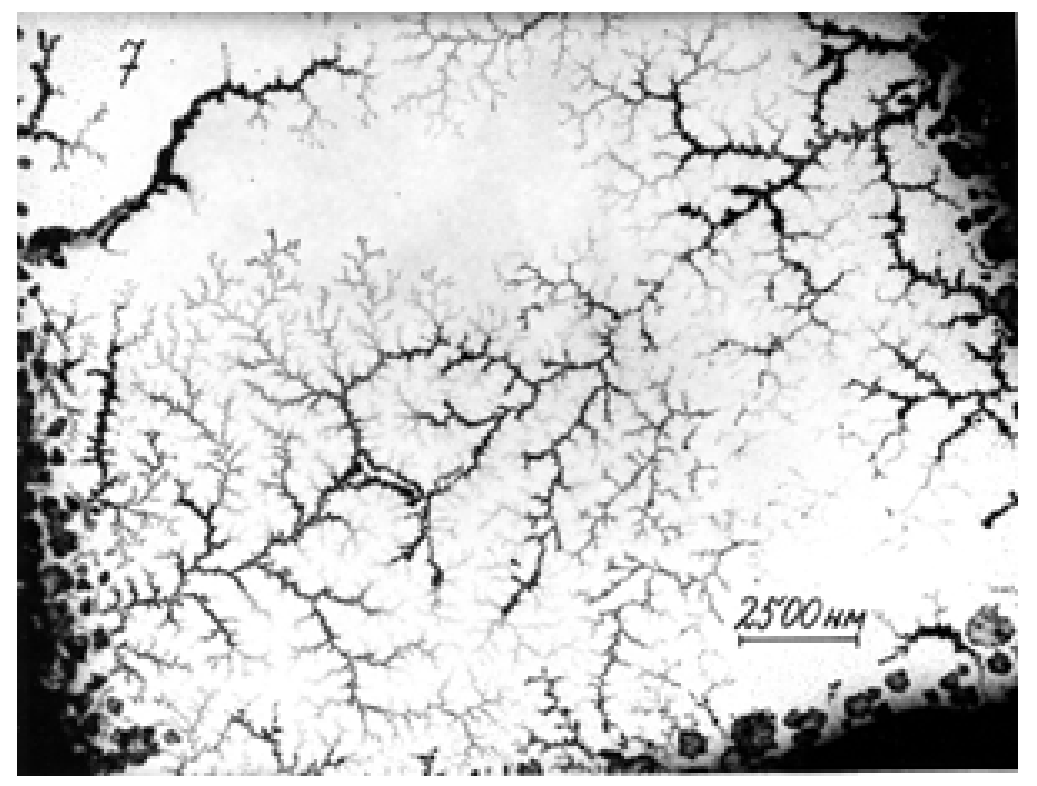

Fig. 3. Dendritic crystal of $\mathrm{AuCl}$ near the boundary of the film

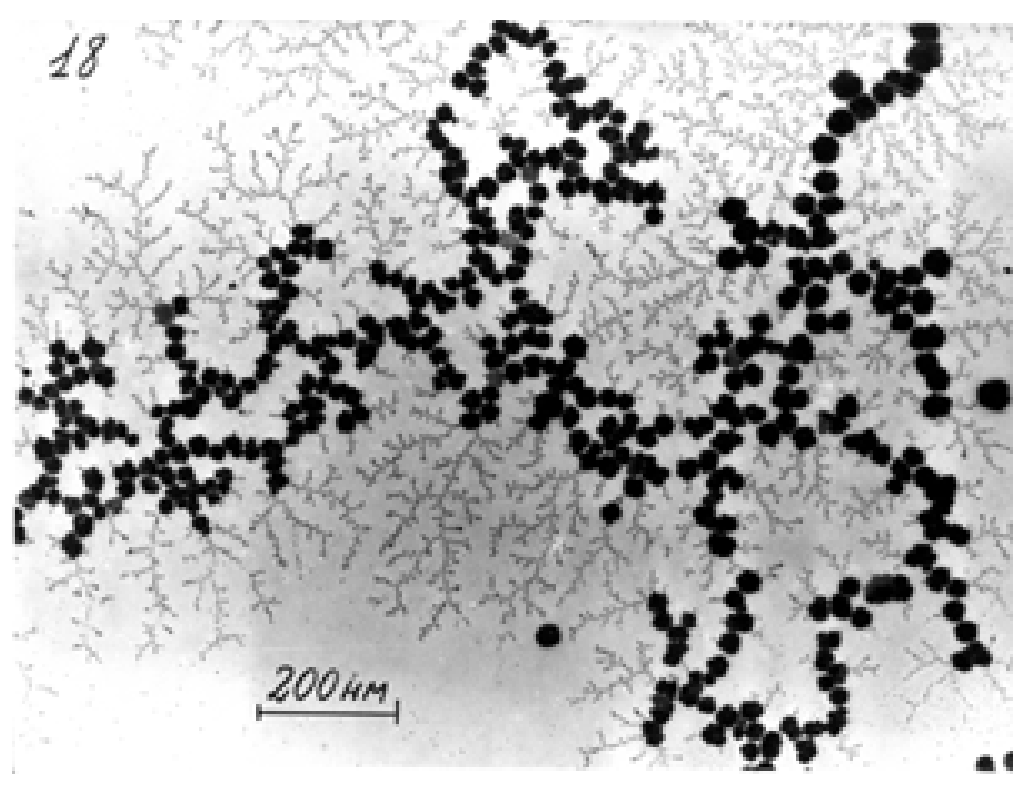

Fig. 4. Point centres and dendritic crystal of $\mathrm{AuCl}$

In a wide range of sizes, such dendritic crystals can be considered as fractal structures. Those fractal dimensions were found by direct calculation using an electron microscope. It was surprising that these dimensions do not coincide for $\mathrm{AuCl}$ and $\mathrm{Cu}$ dendrites, see Fig. 5,6 .

As we can see on Fig. 7, the branches of dendrites consist of microcrystals with diameters $\sim 10 \mathrm{~nm}$. 


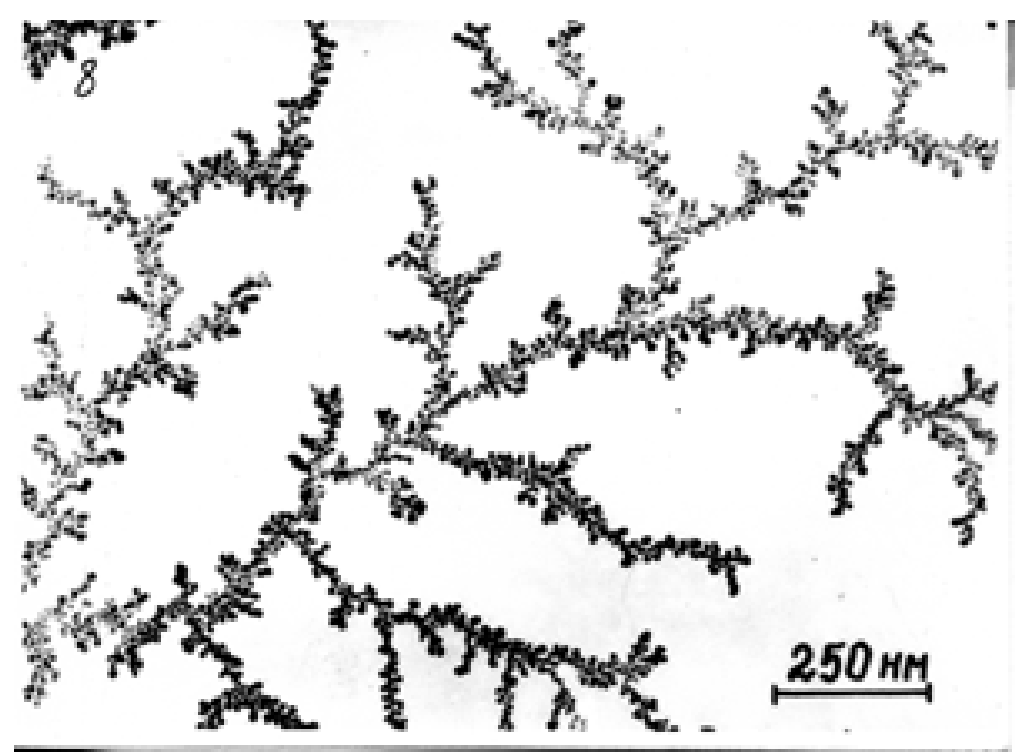

FiG. 5. Dendritic crystal of $\mathrm{AuCl}$, the fractal dimension is $1.63 \pm 0.04$

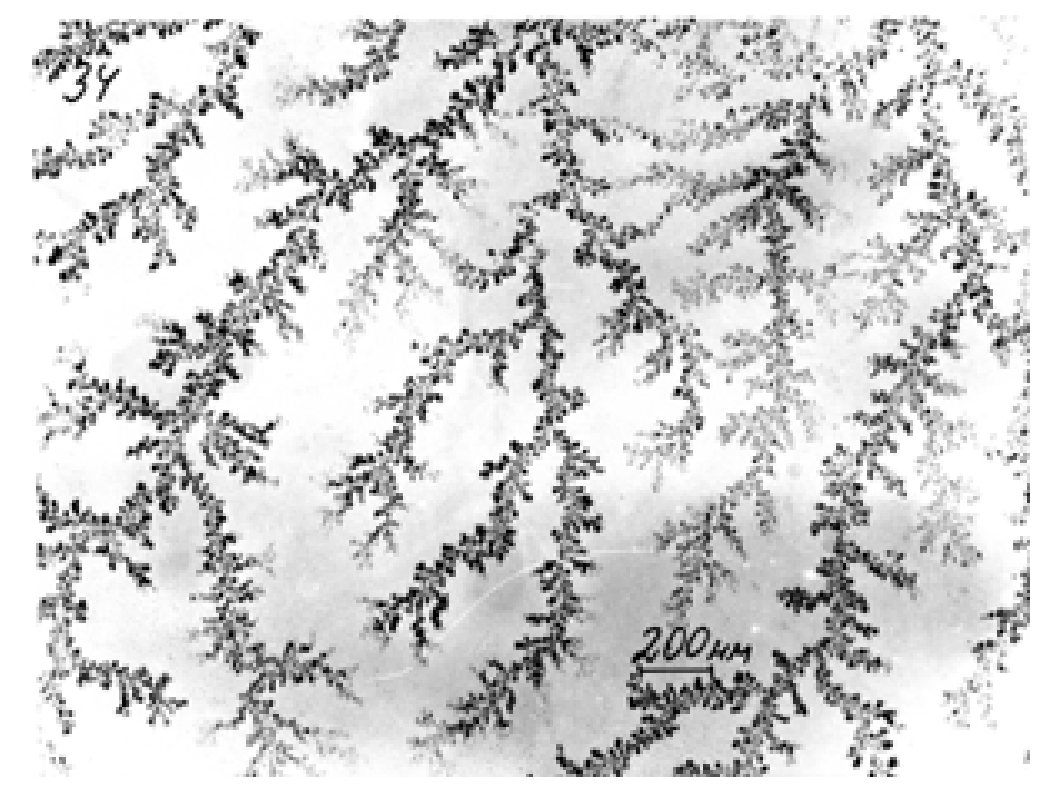

FIG. 6. Dendritic crystal of $C u$, the fractal dimension is $1.76 \pm 0.05$

\section{Evolution of dendrites}

In these experiments, the electron microscope was the source of electrons which caused the decomposition of complex ions, and the same microscope was used to observe results of this reaction. Further observation also caused additional irradiation of previously-formed dendrites. As a result, the dissolution of dendritic crystals take place. This process is accompanied by pulsations of crystal density in channels, branches and those parts disappear and restore at the same places repeatedly. At the end of this process, the crystal eventually disappears, Fig. 8 demonstrating its trace as a cavity in the film. 


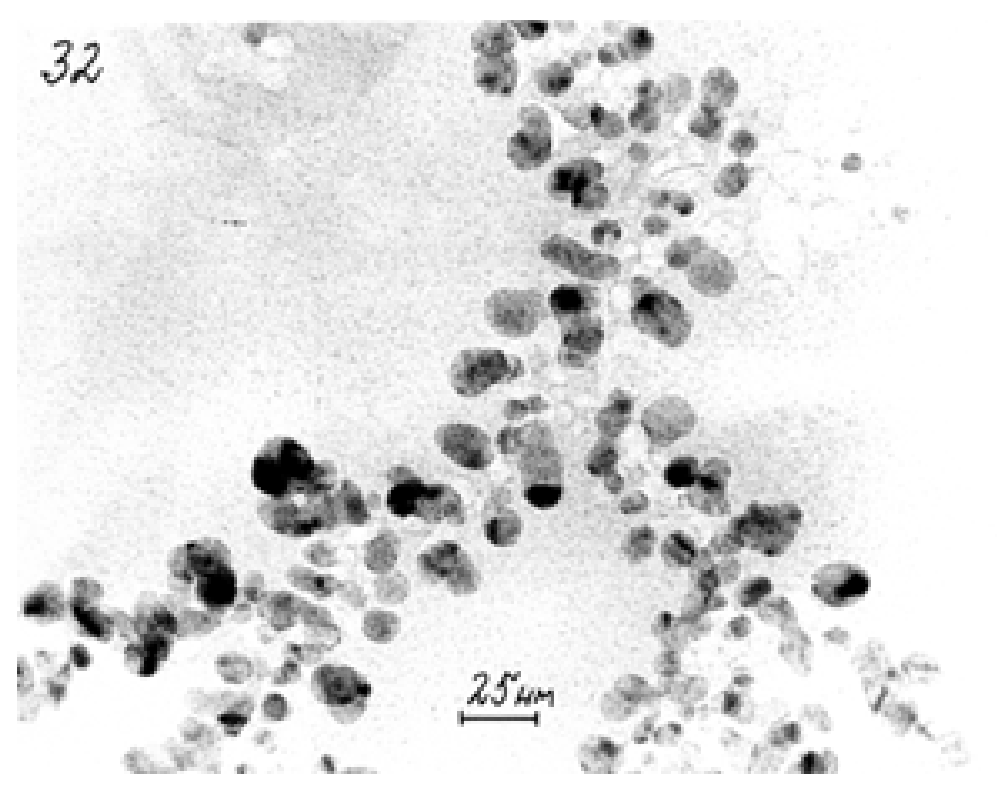

FIG. 7. Microcrystals of $C u$

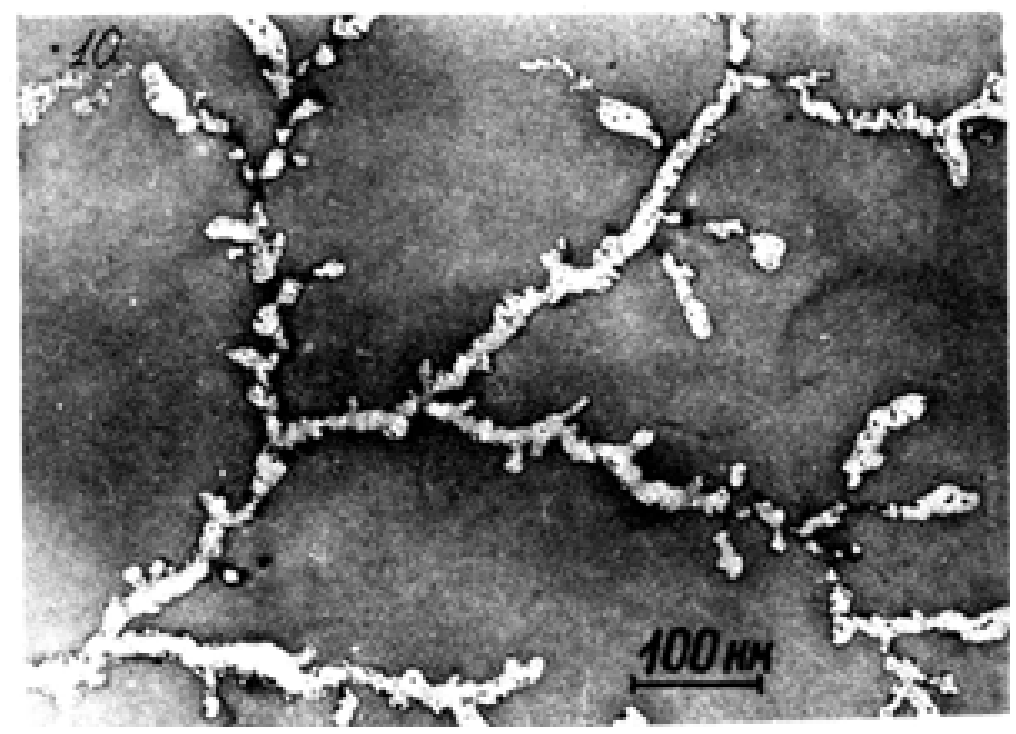

FIG. 8. The cavity at the place of dendritic crystal of $\mathrm{AuCl}$

\section{Conclusion}

In this paper, we only briefly outlined some results of research concerning lightinduced and radiation-induced reactions in compounds of PVA with complex ions of heavy metals. The authors intend to return to problems discussed here and represent them in detail in subsequent publications.

\section{References}

[1] Fedorova A. P. et all. A chain mechanism of radiolysis and photolysis in $H\left[A u C l_{4}\right]$-polyvinyl alcohol system. Radiat. Phys. Chem., 26(3), P. 273-276 (1985).

[2] Plachenov A. B. et all. A chain process of decomposition of halogenide complex ions and the information recording. Bulletin of the Latv. SSR Acad. Sci. Phys. Techn., 5, P. 52-61 (1987) [in Russian] 
[3] Zakharov A. B. et all. Photolysis of the system polyvinyl alcohol - halogen-containing compound of bismuth. J. of Sci. and Appl. Photogr. and Cinem., 32(3), P. 161-167 (1987) [in Russian]

[4] Plachenov A. B. et all. Peculiarities of photostimulated decomposition of the film polyvinyl alcohol $H\left[\mathrm{AuCl}_{4}\right]$ on the boundary of exposed zone (border effect). Russian Journal of Physical Chemistry A, 65(6), P. 1447-1453 (1991) [in Russian]

[5] Plachenov A. B. et all. Radiationally stimulated formation of fractal clusters of $\mathrm{Cu}$ and $\mathrm{AuCl}$. Abstracts of " 8 -th conference on physics and chemistry of nonorganical materials", Tomsk, 1993. II, 75. [in Russian] 